\title{
Critical Appraisal and Proposal of Audit Protocol in-Vogue in India with Respect to Radiation Facilities - A Study Protocol
}

\author{
Anurag A. Luharia, Gaurav Mishra, Bhushan N. Lakhkar and Rajasbala Dhande \\ Department of Radiodiagnosis, Jawaharlal Nehru Medical College, Datta Meghe Institute \\ of Medical Sciences, Sawangi (M), Wardha
}

\section{ABSTRACT}

Radiation has validated its existence and clinical utility in diagnostic and therapeutic applications. At the same time, the risks of radiation that may arise from these applications to the people working in these fields, the general public and the environment are enormous and therefore, need to be assessed and controlled effectively by improving capabilities for controlling hazards, preventing accidents, responding to emergencies and mitigating any harmful consequences of radiation. To critically evaluate the AERB guidelines against the comprehensive audit program given by IAEA for entire radiation facilities and to propose the comprehensive audit program for the institutions / universities. To design the comprehensive audit protocol for national radiation practices at institutional/University level also to propose an upgraded model for accreditation guidelines therein notified by the AERB as against the IAEA comprehensive audit protocol for radiation facilities. The comprehensive audit programs by IAEA the guidelines for the radiation safety and quality assurance by AERB,were compared and evaluated in order to propose the enhanced national audit protocol for the institutions for improving the radiation practicewhich is mainly carried out by the Medical Physicist and Radiological safety officer with the help of Licensee and Employer of the institute / universityon radiation safety and quality for the better streamlined quality and clinical services. Itwill be very useful to improve patient care with the intention of maximizing the effect of clinical care and minimizing its harm to the individual and to society as a whole also patient care, the effective use of resources, the provision and organization of clinical services, professional education and training. The optimized and justified utilization of radiation with radiation dose limitation to radiation worker and the general public in order to minimize the stochastic effect and to avoid the deterministic effect of radiation.

KEY WORDS: ATOMIC ENERGY REGULATORY BOARD (AERB ), INTERNATIONAL ATOMIC ENERGY AGENCY (IAEA), RADIOTHERAPY(RT), RADIODIAGNOSIS(RD), NUCLEAR MEDICINE (NM).

\section{INTRODUCTION}

Application of Ionizing radiation is widely practice all over the world in various domains like research, industries, laboratories and medicine includes radiotherapy, nuclear medicine, radiodiagnosis, medical cyclotron as well as blood irradiators towards the betterment of mankind with specialized consideration of Quality assurance and

Biosc Biotech Res Comm P-ISSN: 0974-6455 E-ISSN: 2321-4007

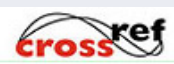

Identifiers and Pagination

Year: 2021 Vol: 14 No (6) Special Issue

Pages: 280-283

This is an open access article under Creative

Commons License Attribn 4.0 Intl (CC-BY).

DOI: $h t t p: / / d x . d o i . o r g / 10.21786 / b b r c / 14.6 .59$
Radiation safety. Even though radiation has proved its existence and effectiveness in the modern medicine where it can be used for detecting and treating malignancy in order to improve survival, on other hand if not used properly it can cause harmful effects to the patients as well as personals who are handling it that's why it is called as double-edged sword. The radiation dose is directly proportional to the risk of developing cancer and at the same time, a subtle balance should be maintained in between the potential benefits and risks involved and it should be noted that the risks may not sway south and outweigh the benefits involved. (Board et al. 2007) This obviates and advocates the need for responsible and acceptable usage of radiation for diagnosis and treatment of concerned disease.

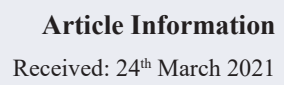

Received: $24^{\text {th }}$ March 2021

ccepted after revision: $2^{\text {th }}$ May 2021 
We are at the dawn of a new revolution/era which is dominated by post-genomic personalized medicine which has techniques based upon physics at the helm of affairs. For the last decade rapid growth in medical radiation application has witnessed in India towards the betterment of mankind, for safe and quality clinical practice, radiation protection and quality assurance. Application of radiation for patient imaging and treatment are very complex kind of procedures, requires lot of precautions and well-trained manpower with skill of radiation handling and safety protections, as per AERB total 349 nuclear medicine facility are registered, 504 radiotherapy facility and around 50 thousand of diagnostic machines are registered.

It will be very difficult task to manage all the radiation facility that's why AERB has approving the nomination of the radiological safety officer and Medical Physicist to take care of quality and radiation safety aspects at each facility with employer and licensee. (Board et al. 2018) AERB has given the guidelines in order to maintain the radiation quality and safety in institution, it will be very useful to have a separate comprehensive audit protocol for each institution in order to meet the expectations of national regulatory bodies also in order to excel at patient care solely intending to maximize the standards of clinical care and at the same time minimising the potential harm rendered to the patient/ individual and also to the whole society.

Rationale: Sudden increase in the radiation facilities strongly encourages us for critically and comprehensively analyze the audit program designed by IAEA and compare with National standard guidelines for designing the comprehensive audit protocols in order to raise the quality standard for safe, justified and optimized radiation practice with maximize the standards of clinical cutting down any potential harm to the individual and to the society.

Aim: To critically evaluate the AERB guidelines from time to time as against the comprehensive audit program given by IAEA with reference to their meaningful applicability to accreditation of radiation facilities and suggesting appropriate inclusions and alterations, if any thereto towards meaningful accreditation of radiation practice at Medical Institutions / Universities including assessing need for a separate autonomous authority / body for exclusively accrediting Medical Institutions / Universities and therein propose an upgraded model of accreditation.

\section{Objectives:}

1. To study the chronological update of the guidelines notified by the AERB as against the notified IAEA comprehensive audit protocol for Radiotherapy, Radio-diagnosis and Nuclear Medicine practice.

2. To identify the inadequacies/limitations, if any, in the updated accrediting guidelines notified by IAEA and AERB with reference to meaningful accreditation of radiation practice at Medical Institutions / Universities.
3. 3.To suggest necessary inclusions and alterations, in the context of identified inadequacies / limitations, in the updated accrediting guidelines notified by IAEA in order to make it feasible for national practice.

4. To propose an upgraded model for accreditation guidelines therein.

5. To design the comprehensive audit protocol for radiation practices at institutional / University level for the implementation.

Research question: Is there a need to critically appraise the national guidelines for radiation facilities (AERB) as against the guidelines laid down by IAEA for all radiation facilities for the purposes of commensuration?

Research hypothesis: There is a need to critically appraise the national guidelines for radiation facilities (AERB)? as against those laid down by IAEA for all radiation facilities for the purposes of commensuration and proposal of an upgraded model thereto.

Null hypothesis: There is NO need to critically appraise the national guidelines for radiation facilities? as against those laid down by IAEA for all radiation facilities for the purposes of commensuration and proposal of an upgraded model thereto.

Alternate hypothesis: There is a need to critically appraise the national guidelines for radiation facilities (AERB)? as against those laid down by IAEA for all radiation facilities for the purposes of commensuration and proposal of an upgraded model thereto.

\section{METHODOLOGY}

\section{Type of study:- Descriptive study}

Duration of study: - 3 years

Place of study: - Datta Meghe Institute of Medical Science (Deemed to be University), Sawangi Meghe, Wardha.

\section{Steps involved:}

1. Procurement guidelines notified by the AERB for the purposes of safe and quality radiation practice from time to time.

2. Procurement guidelines and comprehensive audit program notified by the IAEA for the purposes of safe and quality radiation practice from time to time.

3. Critically analyzing the accrediting guidelines notified by AERB from time to time as against the notified International comprehensive audit program by IAEA.

4. Identifying the inadequacies/ limitations, if any therein with reference to meaningful exclusive accreditation of radiation practices.

5. Suggesting necessary inclusions and alterations, in the context of identified inadequacies/ limitations in order to tide over and make them meaningfully 
relevant for quality and safe radiation practice at institution / Universities.

6. Assess the need of establishment an autonomous exclusive accrediting authority / body for meaningful accreditation of Medical Institutions / Universities.

7. Design the comprehensive audit program at institution / Universities.

Expected Results: It will be very useful to improve patient care with the intention of maximizing the effect of clinical care and minimizing its harm to the individual and to society as a whole also patient care, the effective use of resources, the provision and organization of clinical services, professional education and training. It will not only standardise the radiation facility but also made institution to strictly follow the rules and regulation given by competent authority with reduced quality clinical and technical care.

It will be helpful to capture the actual level of competence of a department, the auditaddresses simultaneously the issues of equipment, infrastructure and operationof clinical practice. A major part of the audit is patient oriented. Therefore, it will also help to streamline the path of clinical services for patients from thediagnosis and the decision to treat, through treatment prescription, planning, treatment preparation and delivery, and then through the follow-up process.Clinical and medical physics procedures include radiation safety and patientprotection when appropriate. Professional training programmes for radiation professional.

\section{DISCUSSION}

(Sonawane et al. 2010) reported that the radiological safety and QA conducted the audit in India including 118 medical X-ray diagnostic installation setup facilities revealed several instances of noncompliance with the requirements of the national safety code; these were addressed and corrected. Some of the major noncompliances were: $\mathrm{kVp}$ calibration inaccuracy $(23 \%)$; noncongruence in between the radiation field and optical field (23\%); non linearity in between the milliamperage station (16\%) \& timer (9\%); faulty knob for adjusting alignment of field size (4\%); improper placement and working of collimator/diaphragm (19.6\%); nonavailability of warning light/ red light just outside the procedure area (29\%); mobile protective barriers being used without proper usage of lead glass viewing window (14\%), etc.

This survey generated awareness about the need and importance of in-house periodic radiological safety and QA audits to ensure optimal radiological protection for patients, occupational workers in diagnostic radiology, and the public. This study makes significant contributions for improving the status of radiological safety of medical $\mathrm{X}$-ray installation setups in India and could provide a vital feedback in reviewing and preparation of regulatory documents pertaining to medical diagnostic X-ray practice.
(Dondi et al. 2017) reported that for an ideal nuclear medicine facility, implementation of Quality Management Services (QMS) should be prioritised with the sole aim of enhancing the overall standards of clinical care provided. The way and manner in which a QMS is designed and implemented is always influenced by multiple needs, constraints, objectives, nature of services, processes used, and the overall size and structure of the nuclear medicine facility. QMSs are expected to be undergoing documentation and regular maintenance in such a way that effectiveness can be perpetually improved in concordance with the stipulated requirements as demanded by the professional, regulatory, and accrediting bodies.

(Quatro et al. 2007)According to IAEA QUANUM program, it has been suggested that the area where the clinical services are being supplied for receiving patients should have the highest level of compliance with stipulated standards. Moreover, issues pertaining to protection of both staff as well as patients from incident as well as scattered radiation demand continuous attention. (Protection et al. 2014)This can be regarded as an important feedback to IAEA with proper regard to the effective ergonomic implementation of Basic Safety Standards recommendations into routine practice.

More focussed training on making standard operating procedures should be availed and due adherence to them should be emphasised when they are accessible. If not accessible, they should be prepared to ensure smoother \& better standardized periodic activities. Implementation of a practice of regular internal audits $\mathrm{Ct}$ when required, follow-up external audits will play a key role in improving radiation protection issues reducing the need for any major investments if need be.As selfassessment program is the one of the most crucial steps of any key audit, QUANUM should also lead towards the introduction of a new methodology which involves selfauditing on a periodic basis and continuous development is ensured.

(Kaur et al. 2013) concluded that audit conducted in radiotherapy, as mentioned by European Commission, "is systematic review of procedures \& practices which are followed. Modifications of practices are directed and implemented where indicated and new standards applied as obviated". The continual and progressive use of radiation therapy and exponential technological developments have necessitated the importance of apt justification, optimization and quality assurance.

(Torras et al. 2017) observed that focussed pointing out and choosing relevant quality indicators is equal to the manner and efficacy in which the clinical audit is designed, implemented and carried out. They reported that their previous audit had reiterated the significance of implementing well-defined procedures for clinical audit. Also, they learnt that selection, relevance of components of the audit team are key to guarantee an audit which is free from bias. They mentioned the need and feasibility of simultaneous audits if need be at multiple sites under 
consideration. They also suggested that such multicentric audits will provide knowledge transferrable and translatable to other national and international centres guiding them to follow in the same footsteps.

They mentioned the outcome of their project as a crucial step towards progressive cooperative and parallel working of radiotherapy processes among multiple radiotherapy facilities. Though immediate unification of all the involved facilitating modalities is still a distant dream, when it comes to diagnostic, treatment related and follow up related aspects at the centres under consideration. Therefore, further comprehensive and minute understanding and emphasis for the need to compare clinical practice at institution under consideration to the practices observed at the institutions which have been already audited methodically. It is true that such endeavours are time-consuming and complex in nature and methodology where the potential benefits totally rely on identifying and rectifying deficiencies in quality control procedures. Clinical audits conducted by relevant third parties or external to the home radiation facility can actually enhance both safety of patient and quality of clinical care.

IAEA has advised the comprehensive clinical audit protocol for the radiology practicing institution to adopt it for quality improvement and learning as well as patient care in order to achieve the aim of diagnostic radiology of minimizing the patient dose with good quality of image. IAEA has also designed the independent comprehensive external audit program for radiotherapy in order to improve the patient set up, patient immobilization, simulation, planning image verification and treatment execution. (Delis et al. 2017) They had received many requests from many countries for performing comprehensive audit program in radiation oncology, their suggested team includes radiation oncologist, medical physicist as an advisor to generate the guidelines for IAEA audit team for application and implementation of audit program under QUATRO. This will further streamline the functioning of the overall working of the radiation facility and also will enhance its work standards as they will be externally accredited by a third party which will reduce any chances of potential bias whatsoever.

Another advantage in this direction is that when there is external accrediting body with a prescribed set of standard criteria, the chances of improvement and overall enhancement of the functioning of the radiation facility in totality increases tremendously. Ultimately it is the hammer which strikes the metal in order to shape it in the desired manner and fashion and make it a piece of art. Similarly, the radiation facility can be analogously compared to the metal here with potential to be a piece of excellent function and the externally accrediting body can be considered here the striking hammer.

\section{CONCLUSION}

It is suggested to have a comprehensive audit program at all institutional level with all due consideration of IAEA and AERB guidelines for radiation safety, quality assurance in order to uplift the clinical and professional services towards the betterment of mankind and to optimized and justified utilization of radiation with dose limitation to radiation worker and the general public in order to minimize the stochastic effect and to avoid the deterministic effect of radiation.

\section{REFERENCES}

Board, A.E., 2018. AERB Safety Guide NO. AERB/ RF-RQTL/SG-1.

Board, A.E.R., 2007. AERB Safety Guide.

Delis, H., Christaki, K., Healy, B., Loreti, G., Poli, G.L., Toroi, P. and Meghzifene, A., 2017. Moving beyond quality control in diagnostic radiology and the role of the clinically qualified medical physicist. Physica Medica, 41, pp.104-108.

Dondi, M., Torres, L., Marengo, M., Massardo, T., Mishani, E., Ellmann, A.V.Z., Solanki, K., Delaloye, A.B., Lobato, E.E., Miller, R.N. and Ordonez, F.B., 2017, November. Comprehensive auditing in nuclear medicine through the international atomic energy agency quality management audits in nuclear medicine program. Part 2: analysis of results. In Seminars in nuclear medicine (Vol. 47, No. 6, pp. 687-693). WB Saunders.

Kaur, J., Mohanti, B.K. and Muzumder, S., 2013. Clinical audit in radiation oncology: Results from one academic centre in Delhi, India. Asian Pacific Journal of Cancer Prevention, 14(5), pp.2829-2834.

Latchoumi, T.P., Ezhilarasi, T.P. and Balamurugan, K., 2019. Bio-inspired weighed quantum particle swarm optimization and smooth support vector machine ensembles for identification of abnormalities in medical data. SN Applied Sciences, 1(10), pp.1-10.

Protection, R., 2014. Safety of Radiation Sources: International Basic Safety Standards. General Safety Requirements Part, 3, p.440.

QUATRO, I., 2007. Comprehensive audits of radiotherapy practices: a tool for quality improvement. Vienna, Austria: IAEA.

Sonawane, A.U., Singh, M., Kumar, J.S., Kulkarni, A., Shirva, V.K. and Pradhan, A.S., 2010. Radiological safety status and quality assurance audit of medical X-ray diagnostic installations in India. Journal of Medical Physics/Association of Medical Physicists of India, 35(4), p.229.

Torras, M.G., Fundowicz, M., Aliste, L., Asensio, E., Boladeras, A.M., Borràs, J.M., Carvalho, L., Castro, C., Deantonio, L., Konstanty, E. and Krengli, M., 2017. Improving radiation oncology through clinical audits: introducing the IROCA project. Reports of Practical Oncology \&t Radiotherapy, 22(5), pp.408-414. 